\title{
Systematic review and meta-analysis of clinical outcomes comparison between different initial dialysis modalities in end- stage renal disease patients due to lupus nephritis prior to renal transplantation
}

\author{
Joel Swai ${ }^{1,2^{*}}$ (D) Xiexiong Zhao ${ }^{3}$, Julie-Raisa Noube ${ }^{4}$ and Gui Ming ${ }^{1 *}$
}

\begin{abstract}
Background: Regarding lupus disease activity, morbidity and survival, limited literature concluded conflicting results when comparing hemodialysis versus peritoneal dialysis as initial renal replacement therapies (RRT) prior to transplantation, in lupus nephritis end-stage renal disease (LN-ESRD) patients. This study was aimed to compare the risks of lupus flares, all-cause infections, all-cause cardiovascular events, and mortality, between hemodialysis versus peritoneal dialysis as initial RRT - modality before renal-transplant in LN-ESRD patients, by systematic review and meta-analysis.
\end{abstract}

Methods: PubMed, EMBASE, and SCOPUS were searched for observational-studies comparing LN-ESRD -patients undergoing hemodialysis (Group1) versus peritoneal-dialysis (Group 2) prior to renal-transplantation, by their risks of lupus flare, all-cause infections, all-cause cardiovascular events, and mortality as outcome measures. Relative-Risks of outcomes between the groups measured overall effects at a 95\% significance level. RevMan 5.3 computer software was used for analysis.

Results: From search, 16 eligible studies reported 15,636 LN-ESRD -patients prior to renal transplantation with 4616 patients on hemodialysis, 2089 on peritoneal dialysis, 280 directly underwent kidney transplantation, 8319 were eliminated with reasons and 332 participants' details were not reported. Hemodialysis group had higher risk of allcause cardiovascular events, Relative-Risk $=1.44$ (Confidence Interval:1.02, 2.04), $p$-Value $<0.05$. With regards to risks for mortality, flare and all-cause infections, there were trends that were not statistically significant ( $p$-Value>0.05).

Conclusion: Except for all-cause cardiovascular events in which peritoneal dialysis is superior to hemodialysis offering better outcomes, both treatment modalities offer more or less similar clinical outcomes as effective initial choices of RRT in LN-ESRD patients prior to renal transplant.

The protocol registration: PROSPERO 2019 CRD42019131600.

Keywords: Renal Dialysis, Lupus nephritis, Kidney failure, chronic, Meta-analysis

\footnotetext{
* Correspondence: joel.swai@hotmail.com; zkbgm@126.com

'Department of Nephrology and Rheumatology, Xiangya Third Hospital,

Central South University, Changsha City, Hunan Province, People's Republic of China

Full list of author information is available at the end of the article
}

(c) The Author(s). 2020 Open Access This article is licensed under a Creative Commons Attribution 4.0 International License, which permits use, sharing, adaptation, distribution and reproduction in any medium or format, as long as you give appropriate credit to the original author(s) and the source, provide a link to the Creative Commons licence, and indicate if changes were made. The images or other third party material in this article are included in the article's Creative Commons licence, unless indicated otherwise in a credit line to the material. If material is not included in the article's Creative Commons licence and your intended use is not permitted by statutory regulation or exceeds the permitted use, you will need to obtain permission directly from the copyright holder. To view a copy of this licence, visit http://creativecommons.org/licenses/by/4.0/. The Creative Commons Public Domain Dedication waiver (http://creativecommons.org/publicdomain/zero/1.0/) applies to the data made available in this article, unless otherwise stated in a credit line to the data. 


\section{Background}

End-stage renal disease (ESRD) is an advanced stage of progressively function-loss of kidneys, commonly characterized by an estimated glomerular filtration rate (eGFR) of lower than $15 \mathrm{ml}$ per minute per 1.73 square meters. ESRD results from an ultimate complication of underlying renal debilitating chronic conditions that could range from systemic diseases such as diabetes, hypertension, inflammatory conditions such as glomerulonephritis and tubulointerstitial nephritis, autoimmune disorders like systemic lupus erythematosus (SLE), genetic disorders including polycystic kidney diseases to chronic urinary tract infections and obstructive conditions [1-4].

Being a systemic disease, SLE manifestations spin from causing mucocutaneous inflammations, neurological symptoms, arthritis, and pancytopenia to multi-organ failures. SLE pathogenesis results from the formation of autoantibodies, activation of serum complements, and deposition of immune complexes in various tissues followed by the initiation of inflammation. The deposition of these immune complexes and their associated inflammations in the kidneys result in lupus nephritis [5]. About $60 \%$ of SLE patients will eventually complicate to lupus nephritis, with male gender and black ancestry in preference $[1,6]$.

About $10 \%$ of all lupus nephritis patients will eventually progress to ESRD which may necessitate RRT such as hemodialysis, peritoneal dialysis and/or kidney transplant [7]. Previous literature has shown that the choice of RRT modality strongly depends on ethnicity [8], employment status, medical insurance type $[8,9]$ and comorbidities burden [10]. Behind diabetes mellitus and hypertension which are by far the major causes of ESRD in adults, by the year 2012, lupus nephritis constituted $1.60 \%$ of all ESRD patients in the United States of America [11]. In children, however, during the 2009-2013 period, cystic and congenital disorders constituted the leading causes of ESRD with 33\% ahead of glomerular disease $(24.60 \%)$, and other secondary causes of glomerulonephritis which constituted about 13\% [12].

Hemodialysis and peritoneal dialysis serve as initial RRT modalities prior to kidney transplantation which is considered a superior modality of the 3 , in terms of patients' survival and quality of life $[13,14]$. On adequate immunosuppressive drugs, 10 to $28 \%$ of LN-ESRD patients on dialysis will improve enough not to require dialysis any further [15]. Therefore, though debatable, a short period of time (i.e. not exceeding 24 months [16]) on dialysis after developing ESRD due to lupus nephritis is advised, before transplantation is opted $[15,17,18]$.

In terms of SLE disease activity and morbidity, a few publications available have concluded conflicting results when comparing hemodialysis versus peritoneal dialysis as initial RRT modalities prior to renal transplantation in LN-ESRD patients. Tsai [19] opposed by Krane [20], reported more SLE disease activity in hemodialysis patients than in peritoneal dialysis, Chang [21] opposed by Kang [13], reported higher risk of infections in hemodialysis than peritoneal dialysis patients, Tsai [19] opposed by Weng [22], reported a higher risk of allcause cardiovascular events in hemodialysis than peritoneal dialysis and Wu [23] opposed by Contreras [24], reported a higher risk of mortality in hemodialysis than in peritoneal group. Ntatsaki et al. [16] in a large study reported similar risks of mortality between the groups.

Therefore, this study will compare between hemodialysis versus peritoneal dialysis modalities in terms of the risks for disease activity, all-cause infection, all-cause cardiovascular events, and mortality in LNESRD adult patients, as initial RRT modality before renal transplant, by systematic review and meta-analysis of available literature.

\section{Methods \\ Study registration}

The protocol for this study was registered at PROSPERO 2019 CRD42019131600 and it can be found via the following link; https://www.crd.york.ac.uk/prospero/display_record.php?RecordID $=131600$

\section{Eligibility criteria}

This study included participants with ESRD (i.e. eGFR of lower than $15 \mathrm{ml}$ per minute per 1.73 square meters) due to lupus nephritis receiving either of the 2 initial RRT namely, hemodialysis or peritoneal dialysis, prior to renal transplant. Both adults (i.e. more or equal to 18 years of age) and pediatric (i.e. less than 18 years old) participants were eligible for inclusion. The main outcomes were; risks of lupus flare, all-cause infections, allcause cardiovascular events and mortality. Both prospective and retrospective conducted matched casecontrol studies comparing the suitable outcomes between the 2 initial dialysis modalities in LN-ESRD were eligible for inclusion. To increase the external validity of this study, accessible literature from all around the world were eligible for inclusion. Only English publications were eligible for inclusion.

\section{Information sources}

The 3 online databases, namely PubMed, EMBASE and the SCOPUS were searched to come up with eligible included studies. The searches were not customized for searching within any restricted date ranges. Secondary referencing of eligible studies was done to extend the search scope. The last date of the search was 28th September 2019. 


\section{The search}

To generate a set of citations that were relevant to our study's search question, an advanced search tool was used in all of the 3 databases aforementioned. Using PubMed, MeSH search builder was utilized; (("Kidney Failure, Chronic" $[\mathrm{MeSH}]$ AND "Renal Replacement Therapy" $[\mathrm{MeSH}]$ ) AND "Renal Dialysis" [MeSH]) AND "Lupus Nephritis"[MeSH] AND "humans"[MeSH Terms]. The search was Repeated with; ((("Lupus Nephritis" [MeSH] AND "Peritoneal Dialysis" [MeSH]) AND "Renal Dialysis"[MeSH]) AND "Kidney Transplantation" [MeSH]) AND "Kidney Failure, Chronic"[MeSH] AND "humans"[MeSH Terms]. Furthermore, a combination of keywords (non-Mesh) was also used to provide more results. These searches were independently performed by 2 authors; JS and XZ. Results were exported to computer software, EndNote X9 (Bld 12,062) which was used to manage and keep track of references throughout this study.

\section{Study selection process}

All studies resulting from the online database search, independently conducted by 2 authors, were screened by their titles and abstracts to initially assess their relevance to our study question. This was, the first-level screening, and was done by the same 2 authors; JS and XZ. Compiled results of first-level screening were then searched for their full-text articles. Second-level scrutiny involved assessing the retrieved full-text articles for eligibility for inclusion or exclusion. Any differences of thoughts in the search process were settled by the third author, JN. The search process is summarized in Fig. 1 .

\section{Data extraction}

Before data was extracted from full-text articles meeting eligibility criteria for inclusion, assessment for methodological biases was done using the Newcastle - Ottawa quality assessment scale [25]. PRISMA (preferred reporting items for systematic reviews and meta-analyses) tool [26] was used for this study write-up to minimize reporting bias.

The process of data extraction was independently performed by 2 authors, namely JS and XZ. Any difference in thoughts was settled by the third author, GM. Data collected included participants' demographics, study characteristics and reported clinical outcomes in line with our study question.

Demographic data included participants' mean age and ethnicity. Modality of dialysis used, whether hemodialysis or peritoneal dialysis and the number of participants undertaking the modality prior to renal transplant were also recorded. Follow-up time and whether a participant switched to another treatment modality during the follow-up period was recorded as well. These participants were later eliminated from the analysis.

In line with this study question, outcomes recorded from the eligible studies included; risks of lupus flare, all-cause infection, all-cause cardiovascular events, and mortality. These outcomes were recorded depending on the treatment group of occurrences i.e. hemodialysis group or peritoneal dialysis group. The risk was defined as the number of participants developing an outcome of interest (i.e. all-cause infection, flare, all-cause cardiovascular event or mortality) during the study follow-up period, divided by total number of participants in the treatment group.

\section{Analysis}

Data were analyzed separately according to the outcomes of interest. This gave rise to 4 separate analyses; comparison of risk of all-cause infections between hemodialysis and peritoneal dialysis groups; comparison of risk of lupus flares between hemodialysis and peritoneal dialysis groups; comparison of risk of all-cause cardiovascular events between hemodialysis and peritoneal dialysis groups; and lastly comparison of risk of mortality between hemodialysis and peritoneal dialysis groups. Risk ratio (RR) was used to measure and compare outcomes modified by the 2 dialysis modalities.

The overall effects of dialysis modalities were diagrammatically depicted by forest-plots. Data synthesis, analysis, and generation of forest-plots were done utilizing computer software, Review Manager (RevMan Version 5.3). The software was customized to a random or fixed effect model depending on the heterogeneity $\left(\mathrm{I}^{2}\right)$ of the studies when analyzing the outcomes. The fixed-effect model was used when $\mathrm{I}^{2}$ was less than $50 \%$ and the random effect model was used if $\mathrm{I}^{2}$ was more than $50 \%$.

\section{Assumptions and simplifications}

For this study purpose, all participants were considered to have been correctly diagnosed with end-stage renal disease strictly due to lupus nephritis and not due to other causes of ESRD such as diabetes or hypertension. Amid 6 guidelines to manage lupus nephritis [27], none is currently specified for LN-ESRD, hence authors assumed that all participants, despite study country had received standard care aligning with internationally accepted guidelines with KDIGO (Kidney Disease- Improving global outcomes - CKD evaluation and management) [28].

\section{Results}

Search

Preliminary search from online databases using a combination of terms in the advanced search tool and $\mathrm{MeSH}$ terms resulted in 302 studies. Of 302 studies, 33 were 


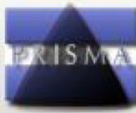

\section{PRISMA 2009 Flow Diagram}
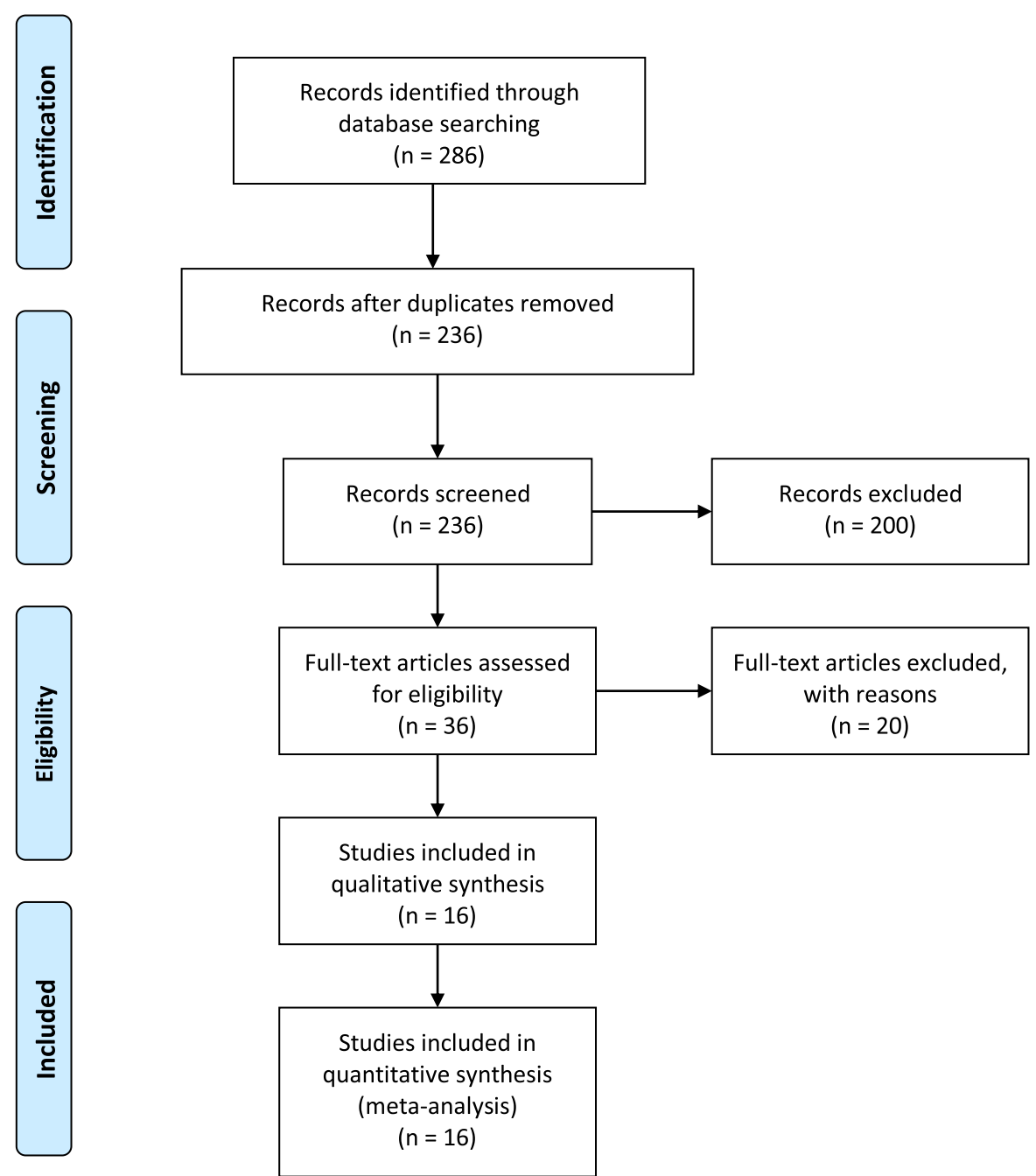

Fig. 1 PRISMA 2009 Flow Diagram for study selection

duplicates, hence discarded. Of 269 remaining studies, 84 were from PubMed, 102 from EMBASE and 83 were from SCOPUS. These were exported to EndNote.

First level scrutiny i.e. screening titles and abstracts, resulted in the elimination of 233 studies as were irrelevant to our study question. Full-text articles for the remaining 66 studies were sought and were screened for inclusion and exclusion criteria i.e. Second level scrutiny. Full-text articles of 3 otherwise eligible studies, [7], [17] and [29] were published in Italian, German and Croatian respectively, hence excluded. A study by Goo et al. [30], was excluded because it did not report the exact number of patients developed our outcome of interests, only reported increase in maximum SLE Disease Activity Index
(SLEDAI) score after RRT and insignificant difference at 2, 5 and 10 year survival between the modalities. After the second scrutiny and elimination of duplicate studies, 16 studies were ultimately eligible for inclusion in this review and meta-analysis.

\section{Study characteristics}

A characteristic summary of 16 articles included in this study is illustrated in Table 1. From 16 ultimately eligible studies, 15,636 patients were diagnosed to have LN-ESRD prior to renal transplantation. Of 15,636 patients, 4616 were on hemodialysis and 2089 were on peritoneal dialysis, 280 directly underwent kidney transplantation, 8319 were eliminated in 1 study [24] after 


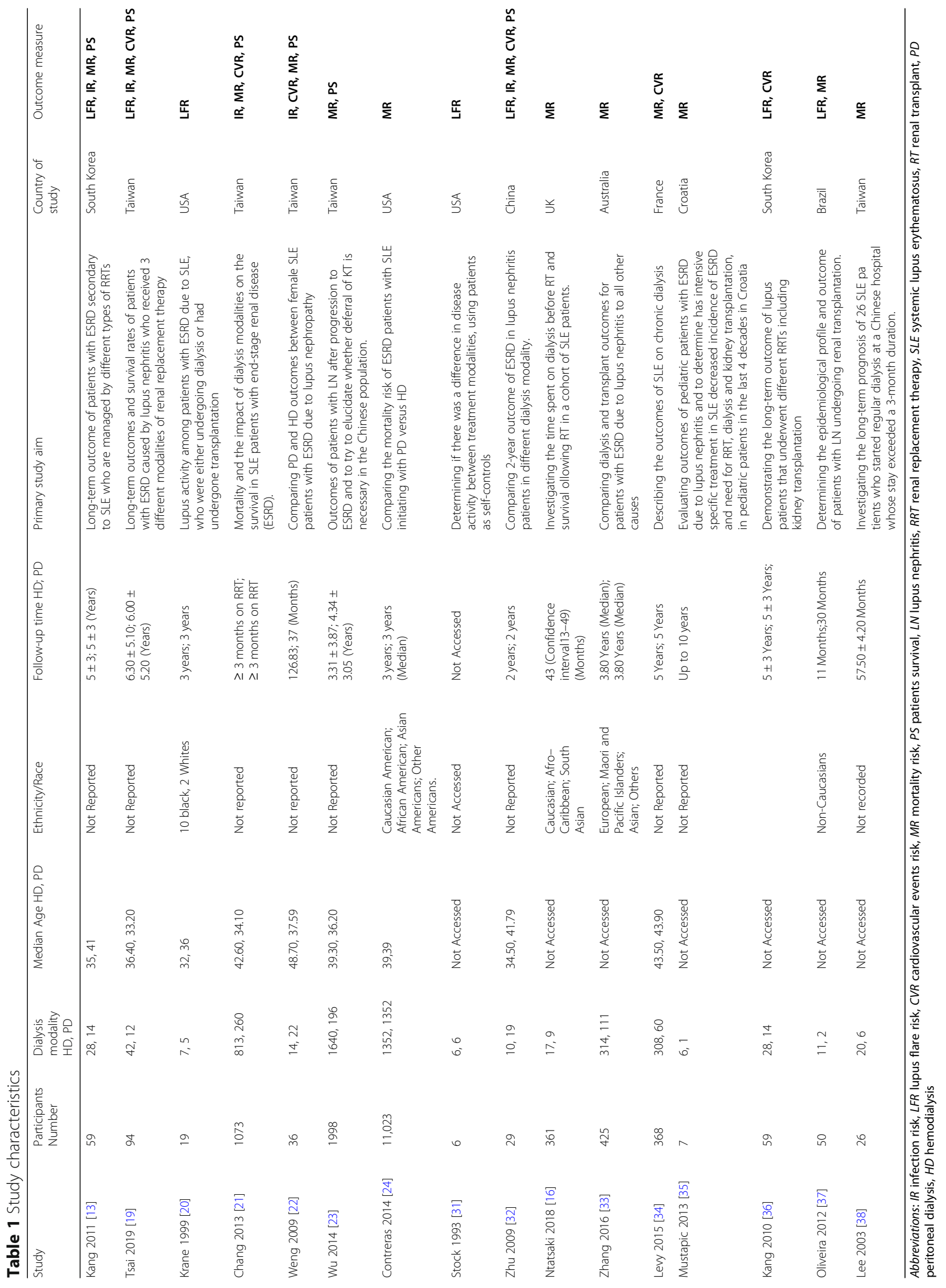


matching participants utilizing propensity scores by the primary author and for 332 participants details were not reported [16].

All 16 studies reported 1 or more outcomes of interest. Studies reporting similar outcomes of interest were analyzed together. A total of 7 studies compared lupus flare risk [13, $19,20,31,36,37$ ] between the 2 dialysis modality groups, 5 compared all-cause infection risk [13, 19, 21, 22, 36], 6 compared all-cause cardiovascular events risk [13, 19, 21, 22, 34, $36]$ and 12 compared the risk of mortality [13, 16, 19, 21$24,33-35,37,38$ ] between the 2 dialysis modality groups.

A total of 5 studies were conducted in Taiwan [19, 21-23, 38], 3 in the United States of America (USA) [20, 24, 31], 1 in China [32], UK, Australia, France, Croatia, Brazil, and 2 in South Korea [13, 36]. All studies were retrospectively conducted studies while $2[22,38]$ were prospectively conducted.

\section{Sources of bias}

All 16 eligible studies included in this study were assessed for risk of bias using the Newcastle - Ottawa quality assessment scale (Table 2). Sample sizes for participants differed from study to study. Other studies had larger sample sizes [24] while other studies had as smaller sample sizes [31]. Larger sample sizes are more likely to represent the general population (i.e. Generalizability) than are smaller sample sizes. Furthermore, none of these 16 eligible studies showed to have calculated required sample sizes prior to conducting the studies.

Table 2 Study bias by Newcastle - Ottawa quality assessment scale

\begin{tabular}{llll}
\hline Study & Selection & Comparability & Exposure \\
\hline Kang 2011 [13] & $\star \star \star$ & $\star$ & $\star \star \star \star$ \\
Tsai 2019 [19] & $\star \star \star$ & $\star$ & $\star \star \star \star$ \\
Krane 1999 [20] & $\star \star \star$ & $\star$ & $\star \star \star \star$ \\
Chang 2013 [21] & $\star \star \star$ & $\star$ & $\star \star \star \star$ \\
Weng 2009 [22] & $\star \star \star$ & $\star$ & $\star \star \star \star$ \\
Wu 2014 [23] & $\star \star \star$ & $\star$ & $\star \star \star \star$ \\
Contreras 2014 [24] & $\star \star \star$ & $\star \star$ & $\star \star \star \star$ \\
Stock 1993 [31] & $\star \star \star$ & $\star$ & $\star$ \\
Zhu 2009 [32] & $\star \star \star$ & $\star$ & $\star \star \star$ \\
Ntatsaki 2018 [16] & $\star \star$ & $\star$ & $\star \star \star \star$ \\
Zhang 2016 [33] & $\star \star \star$ & $\star$ & $\star \star \star \star$ \\
Levy 2015 [34] & $\star \star \star$ & $\star$ & $\star \star \star \star$ \\
Mustapic 2013 [35] & $\star \star \star$ & $\star \star$ & $\star \star \star \star$ \\
Kang 2010 [36] & $\star \star \star$ & $\star$ & $\star \star \star \star$ \\
Oliveira 2012 [37] & $\star \star$ & $\star$ & $\star \star \star \star$ \\
Lee 2003 [38] & $\star \star \star$ & $\star$ & $\star \star \star \star$ \\
\hline \multirow{2}{*}{ Score } & & $\star \star \star \star$
\end{tabular}

$\star$ - Score
Except for Contreras [24], who matched the 2 comparison groups by age, 15 other studies used different mean-aged groups to compare outcomes in hemodialysis versus peritoneal dialysis groups. Furthermore, 2 same comparison groups, say hemodialysis group, from 2 different studies, had different mean-age of their participants. This reduces the comparability of the studies and increases heterogeneity.

Both retrospective [13, 21] and prospective study [22] designs were found to be eligible for inclusion in this study. Generally, prospectively conducted studies have fewer chances of bias and confounders as compared to retrospectively conducted studies. Prospective studies have lesser information and recall bias risks for bias than retrospective studies. On the other hand, retrospective studies have lesser attrition bias risks. All studies reported having used American College of Rheumatology criteria to diagnose SLE hence low selection biases. Furthermore, the dialysis modality utilized was obtained from patients' records hence mitigating selection biases. However, despite a number of factors reported to be influencing the selection of either HD or PD $[9,39]$, physicians' clinical judgment could be playing a role as a clinically sicker patient is likely to be administered more aggressive approaches as HD than PD, inducing selection biases.

Sampling participants from various countries could be beneficial in increasing generalizability but on the other hand, could mean different treatment guidelines or access to healthcare hence reduce comparability. Also, of all 16 studies, none is from a country from Africa thus less representative of the world's population. This creates bias as different ethnicities have also been reported to have different clinical outcomes [11] in regards to SLE outcomes, with Black having the worst course than Caucasians.

Despite the fact that all participants had ESRD due to lupus nephritis, 3 [21, 23, 24] studies reported having had LN-ESRD participants with other comorbidities. Different comorbidities in different participants are sources of biases and reduce comparability. Furthermore, comparing all-cause infection risk between the 2 groups could be confounded by different doses of immunosuppressive drugs among participants as per their SLE disease severity. The higher the burden of immunosuppressive drugs would mean the higher the probability of infections.

\section{Lupus flare risk}

Figure 2 illustrates 7 of 16 studies that reported risk of lupus flares in LN-ESRD undergoing hemodialysis and those undergoing peritoneal dialysis. In hemodialysis group flares risk ranged from 0.18 [37] to 1 [20]. In peritoneal dialysis group, flare risk ranged from 0 [19] to 1 
[20]. The overall risk ratio (RR) of lupus flare between the 2 groups was 1.23 (Confidence Interval: 0.82, 1.85). The difference that hemodialysis is associated with more lupus flares, did not reach statistical significance $(P$ value $=0.31$ ). Fixed-effect model was used since heterogeneity, $\mathrm{I}^{2}$, was $0 \%$ (i.e. $\mathrm{I}^{2}<50 \%$ ).

\section{All-cause cardiovascular events risk}

Figure 3 illustrates 6 of 16 studies that reported risk of allcause cardiovascular events in LN-ESRD undergoing hemodialysis and peritoneal dialysis. In hemodialysis group, risk of all-cause cardiovascular events ranged from 0.04 [21] to 0.45 [19]. In the peritoneal dialysis group, risk of all-cause cardiovascular events ranged from 0.25 [21] to 0.16 [19]. The overall risk ratio between the groups was 1.44 (Confidence Interval: 1.02, 2.04). The difference that hemodialysis is associated with more all-cause cardiovascular events than peritoneal dialysis, reached statistical significance $(P$-value $=0.04)$. A fixed-effect model was used since heterogeneity, $\mathrm{I}^{2}$, was $25 \%$ (i.e. $\mathrm{I}^{2}<50 \%$ ).

\section{All cause infection risk}

Figure 4 illustrates 5 of 16 studies that reported risk of allcause infections in LN-ESRD undergoing hemodialysis and peritoneal dialysis. From hemodialysis group, infection rate ranged from 0.09 [21] to 0.46 [13]. From peritoneal dialysis group, all-cause infection risk ranged from 0.07 [21] to 0.79 [13]. The overall risk ratio between the 2 groups was 1.02 (Confidence Interval: $0.66,1.59$ ). This difference that peritoneal dialysis is associated with lesser allcause infection risk, did not reach statistical significance $(P$-value $=0.92)$. A random-effect model was used since heterogeneity, $\mathrm{I}^{2}$, was $53 \%$ (i.e. $\mathrm{I}^{2}>50 \%$ ).

\section{Mortality risk}

Figure 5 illustrates 11 of 16 eligible studies that reported risk of mortality of LN-ESRD patients undergoing hemodialysis and those undergoing peritoneal dialysis. In hemodialysis group, the risk ranged from 0.07 [22] to 0.58 [23]. In peritoneal dialysis group, mortality risk ranged from 0.13 [21] to 0.29 [13]. The overall risk ratio between the 2 groups was 1.29 (Confidence Interval:
$0.95,1.75)$. The difference that hemodialysis is associated with higher risk of mortality than peritoneal dialysis, did not reach statistical significance $(\mathrm{P}$-value $=0.10)$. A random-effect model was used since heterogeneity, $\mathrm{I}^{2}$, was $76 \%$ (i.e. $\mathrm{I}^{2}>50 \%$ ).

\section{Sensitivity analysis}

Following a high heterogeneity observed in analyzing risk of mortality, we attempted to eliminate 1 peculiar study, Mustapic et al. (2013), whom unlike others, assessed pediatric patients. However, the statistical significance on mortality did not change significantly. The newly obtained overall risk ratio was 1.29 (Confidence Interval: $0.95,1.76), \mathrm{I}^{2}=78 \%, p$-Value $=0.10$.

\section{Discussion}

Many studies have compared clinical outcomes between LN-ESRD patients versus ESRD patients due to other causes like diabetes and hypertension. Many have also compared clinical outcomes between different RRT in ESRD due to various causes. Only a few studies have compared clinical outcomes of different RRT in lupus nephritis-caused ESRD. Furthermore, of few studies comparing the RRT in lupus nephritis-caused ESRD, conflicting results about their clinical outcomes have been reported. Our study compared patients' clinical outcomes between LN-ESRD undergoing hemodialysis versus LN-ESRD undergoing peritoneal dialysis prior to a kidney transplant.

From our study, hemodialysis was associated with higher lupus flare risk than peritoneal dialysis, $R R=1.23$ (Confidence Interval: 0.82, 1.85) but the difference did not reach statistical significance $(P$-value $=0.31), \quad P>0.05$. Hemodialysis was associated with higher all-cause infection risk than peritoneal dialysis, 1.02 (Confidence Interval: $0.66,1.59)$ but the difference did not reach statistical significance, $(P$-value $=0.92), P>0.05$. Hemodialysis was associated with higher risk of all-cause cardiovascular events than peritoneal dialysis, 1.44 (Confidence Interval: $1.02,2.04)$ and the difference reached statistical significance, $(P$-value $=0.04) . P<0.05$. Hemodialysis was associated with higher risk of mortality, 1.29 (Confidence

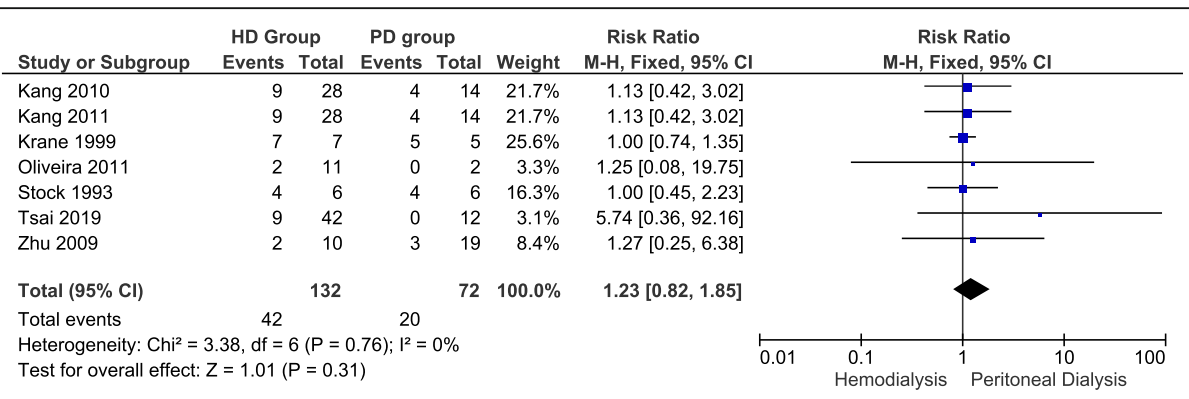

Fig. 2 Risk of lupus flares in LN-ESRD undergoing hemodialysis and those undergoing peritoneal dialysis 


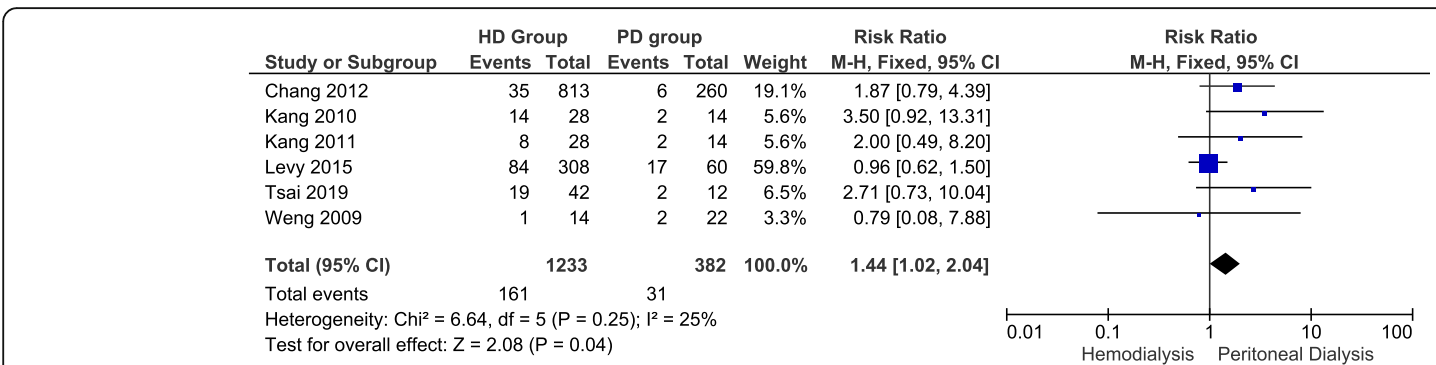

Fig. 3 All-cause cardiovascular events in LN-ESRD undergoing hemodialysis and peritoneal dialysis

Interval: $0.0 .95,1.75)$ than peritoneal dialysis but the difference did not reach statistical significance, $(P$-value $=$ $0.10), P>0.05$. From these results, despite statistical insignificance of all-cause infection risk, lupus flare risk, and mortality, peritoneal dialysis in LN-ESRD is superior to hemodialysis as an initial RRT of choice prior to renal transplant, in terms of better cardiovascular outcomes.

Higher risk of all-cause cardiovascular events in hemodialysis group aligns with contemporary literature that it is accounted for by thrombotic events, vein injury, fibrosis and stenosis associated with central vein access devices such as dialysis catheters [40-42]. The statistically insignificant differences in risk of all-cause infections between the 2 groups could be accounted for by the fact that both modalities are associated with dialysis devices induced infections [43-47]; peritoneal dialysis with peritonitis $[48,49]$ and hemodialysis with central vein access-devices infections $[41,42]$. Statistical insignificant difference between the risk of lupus flares in the 2 comparison groups could be explained by the fact that SLE activity undergoes quiescence, "burn out", when a lupus nephritis patient progresses to ESRD $[15,18]$ and during RRT as shown by Gonzalez-Pulido et al. (2014) [50], ideally due to immunosuppressants administration as illustrated by Maroz et al. (2013) [51]. A small study, by Althaf et al. (2014) [52] however, reported that the activity of SLE could exacerbate, preferably to lupus nephritis if the patient becomes pregnant. Regarding mortality, a study by Mustapic et al. (2013, 36), have reported more deaths to be associated with hemodialysis than peritoneal dialysis, specifically due to cardiovascular events.

According to our study, however, the mortality difference did not reach statistical significance. On the other hand, from our study, PD showed statistically significant benefits in terms of all-cause cardiovascular outcomes as compared to its counterpart group. This is supported by Kang et al. (2010) [36] who concluded the superiority of PD over HD. In another study, Sekkarie [53] shows no recovery advantage for patients treated by peritoneal dialysis as compared with hemodialysis, but in the same study, it was concluded that peritoneal dialysis preserves residual renal function better than hemodialysis.

The results of this study should be interpreted with caution. This is because of possible sources of biases observed at individual studies level as well as this review level. Other included studies used larger sample sizes while others used smaller sample sizes and none of the studies calculated sample sizes and power, thus introducing chances of type-1 error [54-56]. Participants had different mean age groups and some studies reported different comorbidities in their participants. Study settings were also different among included studies with 5 conducted in Taiwan, 3 in the United States of America, 2 in South Korea and 1 from China, Australia, Brazil, and the UK each. These different settings could be advantageous but could also mean different economical levels, different advancements in healthcare facilities.

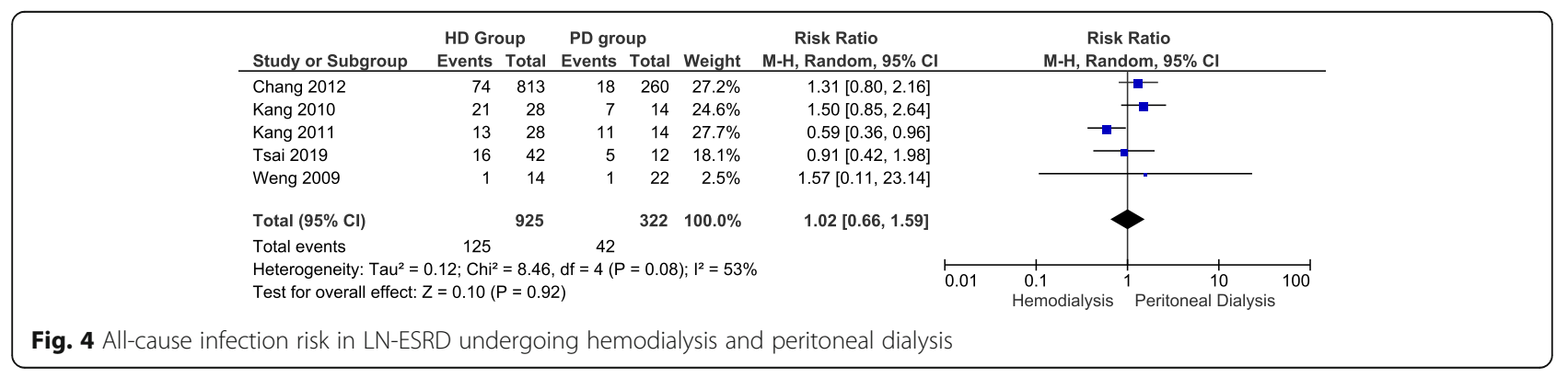




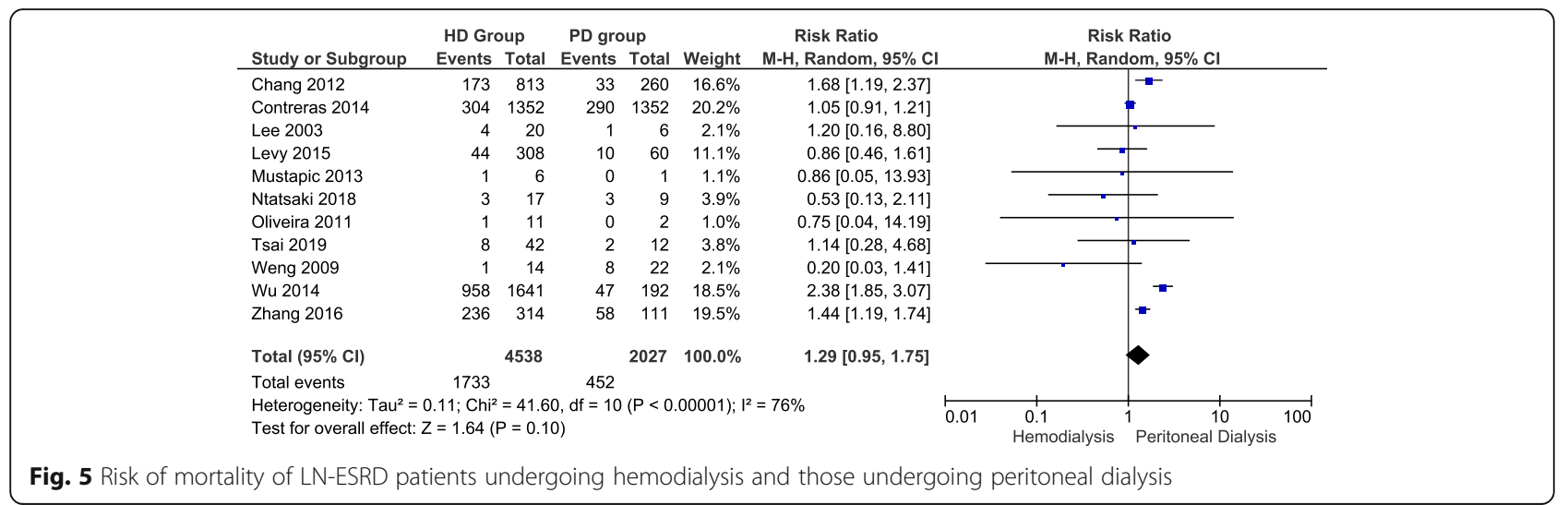

Furthermore, none of the studies was from Africa, thus reduced generalizability. A total of 2 studies, though fulfilling inclusion criteria were prospectively conducted while others were retrospectively conducted. Prospective studies could have lower chances of bias than retrospective studies $[57,58]$.

\section{Conclusion}

Except for all-cause cardiovascular events in which PD is superior to hemodialysis offering better outcomes, both treatment modalities offer more or less similar clinical outcomes as effective initial choices of RRT in LN-ESRD patients prior to renal transplant. We, however, encourage further research on the question addressing better the possible sources of biases encountered in this study.

\section{Abbreviations}

eGFR: Estimated glomerular filtration rate; ESRD: End-stage renal disease; LNESRD: Lupus nephritis end-stage renal disease; SLE: Systemic lupus erythematosus; SLEDAI: Systemic lupus erythematosus disease activity index; RRT: Renal replacement therapies; PRISMA: Preferred reporting items for systematic reviews and meta-analyses; KDIGO: Kidney disease- improving global outcomes; CKD: Chronic kidney disease; MeSH: Medical subject headings; HD: Hemodialysis; PD: Peritoneal dialysis; IR: Infection risk; LFR: Lupus flare risk; CVR: Cardiovascular events risk; MR: Mortality risk; PS: Patients survival; JS: Joel Swai (Author); XZ: Xiexiong Zhao (Author); JN: Julie-Raisa Noube (Author); GM: Gui Ming (Author)

\section{Acknowledgements}

Authors acknowledge supervisory input from Dr. Alphonce Chandika, Executive Director, Benjamin Mkapa Hospital, Dodoma city, Tanzania, East Africa. Authors also acknowledge English language proofreading by Dr. Hu Zixuan; Department of Gastroenterology, Xiangya Third Hospital, Central South University, Changsha City, Hunan Province, Peoples' Republic of China.

\section{Authors' contributions}

Study designing: JS; data search JS and XZ; data extraction: JS, XZ, and JN; data analysis and interpretation: JS and GM; Manuscript drafting: JS; manuscript critical intellectual content revision: GM and JN. All authors read and approved the final version of the manuscript.

\section{Funding}

No funds were given.

\section{Availability of data and materials}

The datasets used and analyzed during the current study are available from the corresponding author on reasonable request.
Ethics approval and consent to participate

Not applicable.

\section{Consent for publication}

Not applicable.

\section{Competing interests}

Authors declared no competing interests.

\section{Author details}

${ }^{1}$ Department of Nephrology and Rheumatology, Xiangya Third Hospital, Central South University, Changsha City, Hunan Province, People's Republic of China. ${ }^{2}$ Department of Nephrology, Benjamin Mkapa Hospital, Dodoma City, Dodoma Region, United Republic of Tanzania. ${ }^{3}$ Department of Cardiology, Xiangya Third Hospital, Central South University, Changsha City, Hunan Province, People's Republic of China. ${ }^{4}$ Department of

Gastroenterology, Xiangya Third Hospital, Central South University, Changsha City, Hunan Province, People's Republic of China.

Received: 25 November 2019 Accepted: 16 April 2020

Published online: 01 May 2020

\section{References}

1. Choi J, Kim ST, Craft J. The pathogenesis of systemic lupus erythematosusan update. Curr Opin Immunol. 2012;24(6):651-7.

2. Finzel S, Schaffer S, Rizzi M, Voll RE. Pathogenesis of systemic lupus erythematosus. Z Rheumatol. 2018;77(9):789-98.

3. Klein A, Polliack A, Gafter-Gvili A. Systemic lupus erythematosus and lymphoma: incidence, pathogenesis and biology. Leuk Res. 2018;75:45-9.

4. Jacobson HR. Chronic renal failure: pathophysiology. Lancet. 1991;338(8764): 419-23.

5. Cameron JS. Lupus nephritis. J Am Soc Nephrol. 1999;10(2):413-24.

6. Gõmez-Puerta JA, Feldman CH, Alarcõn GS, Guan H, Winkelmayer WC, Costenbader $\mathrm{KH}$. Racial and ethnic differences in mortality and cardiovascular events among patients with end-stage renal disease due to lupus nephritis. Arthritis Care Res. 2015;67(10):1453-62.

7. Banfi G, Leoni A, Moroni G. Treatment of lupus nephritis associated with end-stage renal disease. G Ital Di Nefrol. 2008;25(Suppl 44):S68-75.

8. Plantinga LC, Drenkard C, Patzer RE, Klein M, Kramer MR, Pastan S, et al. Sociodemographic and geographic predictors of quality of care in United States patients with end-stage renal disease due to lupus nephritis. Arthritis Rheum. 2015;67(3):761-72.

9. Devlin A, Waikar SS, Solomon DH, Lu B, Shaykevich T, Alarcón GS, et al. Variation in initial kidney replacement therapy for end-stage renal disease due to lupus nephritis in the United States. Arthritis Care Res. 2011;63(12): 1642-53.

10. O'Shaughnessy MM, Montez-Rath ME, Lafayette RA, Winkelmayer WC. Patient characteristics and outcomes by GN subtype in ESRD. Clin J Am Soc Nephrol. 2015;10(7):1170-8

11. Sabucedo AJ, Contreras G. ESKD, transplantation, and Dialysis in lupus nephritis. Semin Nephrol. 2015;35(5):500-8.

12. The National Institute of Diabetes and Digestive and Kidney Diseases: Kidney Disease Statistics for the United States, 2016. https://www.niddk.nih. 
gov/health-information/health-statistics/kidney-disease. Accessed 22 Oct 2019.

13. Kang SH, Chung BH, Choi SR, Lee JY, Park HS, Sun IO, et al. Comparison of clinical outcomes by different renal replacement therapy in patients with end-stage renal disease secondary to lupus nephritis. Korean J Intern Med. 2011;26(1):60-7.

14. Mamatov E, Kocaay AF, Koc MA, Celebi ZK, Sengül S, Keven K, et al. Predictive role of neutrophil Gelatinase-associated Lipocaline in donorspecific antibody-positive and donor-specific antibody-negative renal transplant patients. Transplant Proc. 2015;47(6):1688-91.

15. Cheigh JS, Stenzel KH. End-stage renal disease in systemic lupus erythematosus. Am J Kidney Dis. 1993;21(1):2-8.

16. Ntatsaki E, Velo-Garcia A, Vassiliou VS, Salama AD, Isenberg DA. Impact of pre-transplant time on dialysis on survival in patients with lupus nephritis. Clin Rheumatol. 2018;37(9):2399-404.

17. Gawlik A, Floege J. Dialysis and transplantation in patients with lupus nephritis. Nieren- und Hochdruckkrankheiten. 2004;33(2):97-103.

18. Mojcik CF, Klippel JH. End-stage renal disease and systemic lupus erythematosus. Am J Med. 1996;101(1):100-7.

19. Tsai WT, Chang HC, Wang CT, Chiang BL, Lin YT. Long-term outcomes in lupus patients receiving different renal replacement therapy. J Microbiol Immunol Infect = Wei mian yu gan ran za zhi. 2019;52(4):648-53.

20. Krane NK, Burjak K, Archie M, O'Donovan R. Persistent lupus activity in endstage renal disease. Am J Kidney Dis. 1999;33(5):872-9.

21. Chang YS, Liu CJ, Wu TH, Chaou CH, Lin KC, Ou SM, et al. Survival analysis in systemic lupus erythematosus patients on maintenance dialysis: a nationwide population-based study in Taiwan. Rheumatol. 2013;52(1):166-72.

22. Weng CH, Hsu CW, Yu CC, Yen TH, Yang CW, Hung CC. Peritoneal dialysis and hemodialysis in systemic lupus erythematosus patients: comparison of clinical outcomes. Kidney Blood Press Res. 2009;32(6):451-6.

23. Wu MJ, Lo YC, Lan JL, Yu TM, Shu KH, Chen DY, et al. Outcome of lupus nephritis after entering into end-stage renal disease and comparison between different treatment modalities: a nationwide population-based cohort study in Taiwan. Transplant Proc. 2014;46(2):339-41.

24. Contreras G, Pagan J, Chokshi R, Virmani S, Diego JM, Byers P, et al. Comparison of mortality of ESRD patients with lupus by initial dialysis modality. Clin J Am Soc Nephrol. 2014;9(11):1949-56.

25. Wells GA, Shea B, O'Connell D, et al. The Newcastle-Ottawa scale (NOS) for assessing the quality of nonrandomised studies in meta-analyses [webpage on the internet]. Ottawa: Ottawa Hospital Research Institute; 2011. Available from: http://wnw.ohri.ca/ programs/dinical_epidemiology/oxford.asp. Accessed 22 Oct 2019.

26. Moher D, Liberati A, Tetzlaff J, Altman DG, The PG. Preferred reporting items for systematic reviews and meta-analyses: The PRISMA statement. PLoS Med. 2009;6(7):e1000097

27. Wilhelmus S, Bajema IM, Bertsias GK, Boumpas DT, Gordon C, Lightstone L, et al. Lupus nephritis management guidelines compared. Nephrol Dial Transplant. 2015;31(6):904-13.

28. Kidney Disease. Improving Global Outcomes (KDIGO) CKD Work Group. KDIGO 2012 Clinical Practice Guideline for the Evaluation and Management of Chronic Kidney Disease. Kidney Int. 2013;3:1-150.

29. Katalinić L, Eliasson E, Bubić-Filipi L, Kes P, Anić B, Bašić-Jukić N. Renal transplantation in patients with lupus nephritis. Lijec Vjesn. 2014;136(7-8): 219-23.

30. Goo YS, Park HC, Choi HY, Kim BS, Park YB, Lee SK, et al. The evolution of lupus activity among patients with end-stage renal disease secondary to lupus nephritis. Yonsei Med J. 2004;45(2):199-206.

31. Stock GG Jr, Krane NK. Treatment of end-stage renal disease due to lupus nephritis: comparison of six patients treated with both peritoneal and hemodialysis. Adv Perit Dial Conf Perit Dial. 1993;9:147-51.

32. Zhu M, Yan Y, Ni Z, Qian J. Comparison of two-year outcome in maintenance dialysis lupus nephritis patients. Hemodial Int. 2009;13(3):3856.

33. Zhang L, Lee G, Liu X, Pascoe EM, Badve SV, Boudville NC, et al. Long-term outcomes of end-stage kidney disease for patients with lupus nephritis. Kidney Int. 2016;89(6):1337-45.

34. Levy B, Couchoud C, Rougier JP, Jourde-Chiche N, Daugas E. Outcome of patients with systemic lupus erythematosus on chronic dialysis: an observational study of incident patients of the French National Registry 2002-2012. Lupus. 2015;24(10):1111-21.

35. Mustapic Ž, Puretic Z, Slavicek J, Frkovic M, Jelusic M. Outcome of pediatric patients with end stage renal disease secondary to systemic lupus erythematosus in Croatia in the last four decades. Ann Rheum Dis. 2013; 71(3):707.

36. Kang SH, Hwang HS, Hyoung BJ, Lee SY, Jeon YJ, Chung BH, et al. Comparison of clinical outcome according to different renal replacement therapy in end-stage renal disease patients with lupus nephritis. Am J Transplant. 2010;10:520.

37. Oliveira CS, D'Oliveira I, Bacchiega ABS, Klumb EM, Albuquerque EMM, Souza E, et al. Renal transplantation in lupus nephritis: a Brazilian cohort Lupus. 2012;21(5):570-4.

38. Lee PT, Fang HC, Chen CL, Chiou YH, Chou KJ, Chung HM. Poor prognosis of end-stage renal disease in systemic lupus erythematosus: a cohort of Chinese patients. Lupus. 2003;12(11):827-32.

39. Plantinga LC, Patzer RE, Drenkard C, Kramer MR, Klein M, Lim SS, et al. Association of time to kidney transplantation with graft failure among U.S. patients with end-stage renal disease due to lupus nephritis. Arthritis Care Res. 2015;67(4):571-81.

40. E. Cimochowski G, Worley E, E Rutherford W, Sartain J, Blondin J, Harter H. Superiority of the Internal Jugular over the Subclavian Access for Temporary Dialysis 1990. 154-161 p.

41. Oguzkurt L, Tercan F, Torun D, Yildirim T, Zümrütdal A, Kizilkilic O. Impact of short-term hemodialysis catheters on the central veins: a catheter venographic study. Eur J Radiol. 2004;52(3):293-9.

42. Schwab SJ, Quarles LD, Middleton JP, Cohan RH, Saeed M, Dennis WW. Hemodialysis-associated subclavian vein stenosis. Kidney Int. 1988;33(6): 1156-9.

43. Battistella C, Quattrin R, Celotto D, d'Angelo M, Fabbro E, Brusaferro S, et al. Factors predicting influenza vaccination adherence among patients in dialysis: an Italian survey. Human Vacc Immunother. 2019;15(10):2434-9.

44. Bieber S, Mehrotra R. Peritoneal Dialysis access associated infections. Adv Chronic Kidney Dis. 2019;26(1):23-9.

45. Soi V, Daifi C, Yee J, Adams E. Pathophysiology and treatment of hepatitis B and $C$ infections in patients with end-stage renal disease. Adv Chronic Kidney Dis. 2019;26(1):41-50.

46. Viron C, Lobbedez T, Lanot A, Bonnamy C, Ficheux M, Guillouet S, et al. Simultaneous Removal And Reinsertion of the PD Catheter in Relapsing Peritonitis. Perit Dial Int. 2019;39(3):282-8.

47. Sires RL, Adler SG, Louie JS, Cohen AH. Poor prognosis in end-stage lupus nephritis due to nonautologous vascular access site associated septicemia and lupus flares. Am J Nephrol. 1989;9(4):279-84.

48. Holley JL, Piraino BM. Complications of Peritoneal Dialysis: Diagnosis and Management. Semin Dial. 1990;3(4):245-8.

49. Althaf MM, Abdelsalam MS, Alsunaid MS, et al. Brevibacterium casei isolated as a cause of relapsing peritonitis. Case Reports. 2014;2014:bcr2014203611

50. González-Pulido C, Croca S, Abrol E, Isenberg DA. Long-term activity index after renal failure in a cohort of 32 patients with lupus nephritis. Clin Exp Rheumatol. 2014;32(3):301-7.

51. Maroz N, Segal MS. Lupus nephritis and end-stage kidney disease. Am J Med Sci. 2013;346(4):319-23.

52. Althaf MM, Abdelsalam MS, Alfurayh Ol. Lupus flares in two established end-stage renal disease patients with on-line hemodiafiltration during pregnancy - case series. Lupus. 2014;23(9):945-8.

53. Sekkarie MA, Port FK, Wolfe RA, Guire K, Humphrys R, Van Amburg G, et al. Recovery from end-stage renal disease. Am J Kidney Dis. 1990;15(1):61-5.

54. Blaizot S, Herzog SA, Abrams S, Theeten H, Litzroth A, Hens N. Sample size calculation for estimating key epidemiological parameters using serological data and mathematical modelling. BMC Med Res Methodol. 2019;19(1):51.

55. Nordgren R. Calculating a sample size for a study with repeated measures. J Mol Cell Cardiol. 2019;133:217-9.

56. Raseta M, Bazarova A. Comments on "a general approach for sample size calculation for the three-arm 'gold standard' non-inferiority design". Stat Med. 2019;38(7):1300-2.

57. Blome C, Augustin M. Measuring change in quality of life: bias in prospective and retrospective evaluation. Value Health. 2015;18(1):110-5.

58. Kraft P, Thomas DC. Bias and efficiency in family-based genecharacterization studies: conditional, prospective, retrospective, and joint likelihoods. Am J Hum Genet. 2000;66(3):1119-31.

\section{Publisher's Note}

Springer Nature remains neutral with regard to jurisdictional claims in published maps and institutional affiliations. 